\title{
Agtech infrastructure for pandemic preparedness
}

To the Editor - The world has witnessed multiple outbreaks of epidemic and pandemic disease over the past 20 years, including four outbreaks of Ebola virus in Africa, an outbreak of Zika virus in South America, and three outbreaks of respiratory infections caused by novel $\beta$-coronavirus strains, including the latest (SARS-CoV-2) being responsible for the current COVID-19 pandemic ${ }^{1-3}$. At the time of writing, more than 17 million confirmed SARS-CoV-2 infections have been reported worldwide and nearly 700,000 people have died from the disease ${ }^{3}$. Some of the common themes emerging from these outbreaks include the lack of preparedness of the regional, national and global health authorities, insufficient supplies of personal protective equipment (PPE), and insufficient hospital capacity to address the sudden influx of patients with acute disease. This was exacerbated by the scarcity of reagents, personnel and infrastructure for population-wide testing, diagnosis, and essential track-and-trace strategies to reduce $R$, the effective reproduction number ${ }^{4}$. Here, we note how approaches used in agtech to genotype germplasm could be adapted to testing in the context of a pandemic.

\section{Strategies to stretch and conserve resources}

For localized outbreaks and epidemics, health authorities with sufficient financial resources can turn to outside providers for help to address local shortages of personnel, equipment and reagents. However, the current COVID-19 pandemic has resulted in a concurrent demand for the same resources across the planet. Stockpiles were rapidly exhausted in many places, as predicted by earlier studies of actual and hypothetical outbreaks of novel influenza virus strains $s^{5,6}$, leaving governments scrambling to replenish essential items. In some cases, there has been effective resource allocation and sharing according to need, but there have also been examples of countries attempting to ring-fence supplies or resources at the expense of others, especially for medical apparatus, PPE and reagents such as testing kits ${ }^{7}$.

The pandemic has also shown how flexibility can help to relieve supply bottlenecks. A sudden outbreak of disease leads to the rapid depletion of stockpiles of available equipment and reagents,
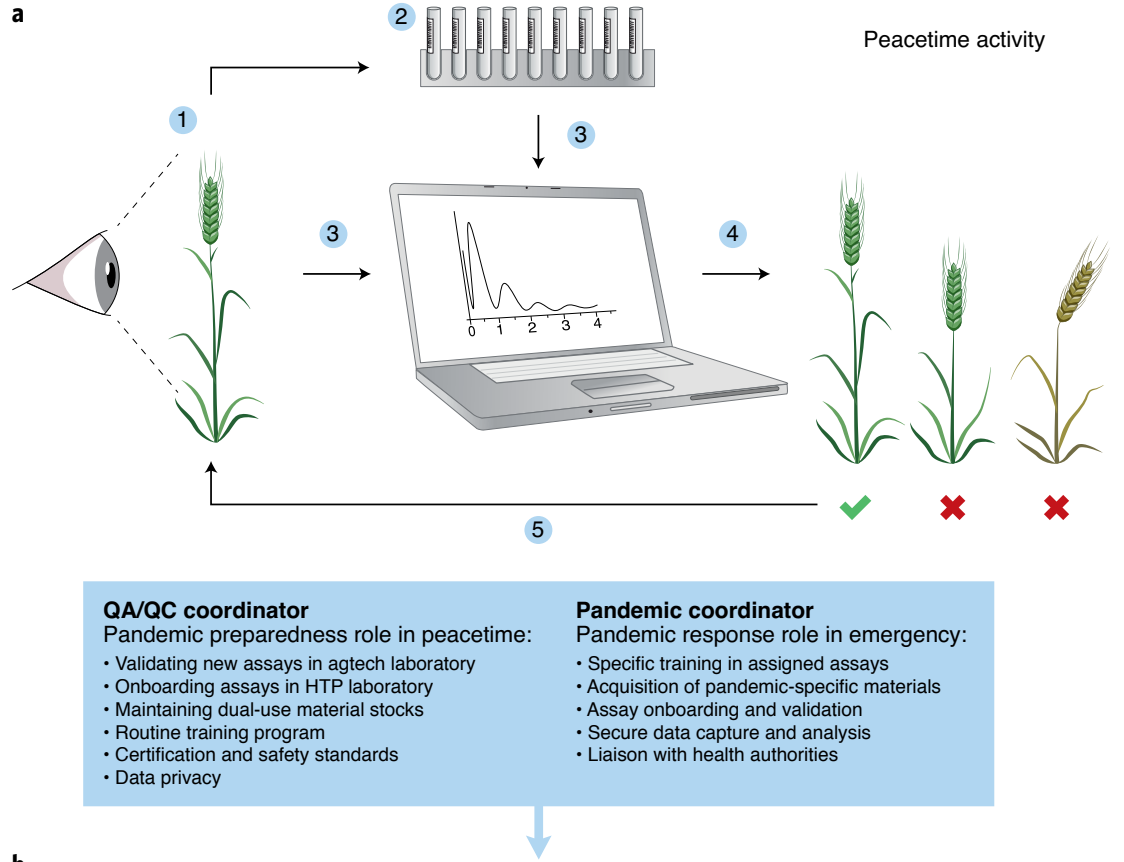

b

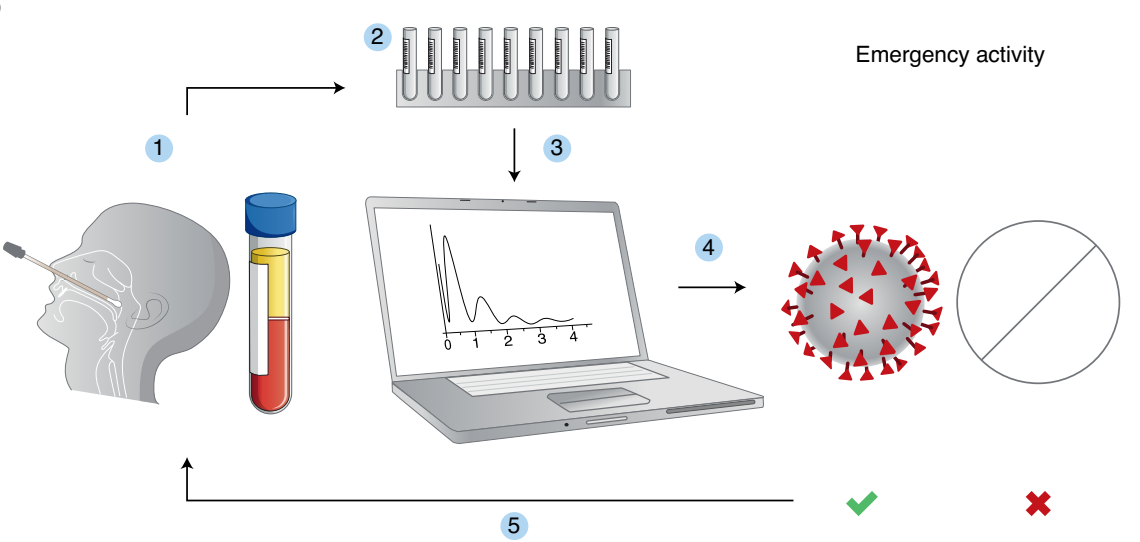

Fig: 1 | Agtech infrastructure and pandemic preparedness. a, Scheme, as exemplified by the GIFS OPAL, routinely used for the genotypic and phenotypic analysis of plants for crop breeding and surveillance for crop diseases. $\mathbf{b}$, Adaptation of agtech process for diagnostics and tracing in a human pandemic. The response would be carried out under the leadership of a peacetime QA/QC coordinator with a dual role as pandemic coordinator in times of national or international emergency. (1) Image capture (remote or in-field sensors) would be replaced by the acquisition of patient samples (here shown as nasopharyngeal swab or blood). (2) Samples for molecular analysis (DNA, RNA or proteins). (3) Data capture and high-throughput analysis. (4) Automated analysis of new cultivars would be replaced by the automated detection of viral RNA or proteins (or detection of neutralizing antibodies). (5) Selection of fittest cultivars for breeding would be replaced by the confirmation of patient diagnoses.

particularly single-use items such as PPE and testing kits ${ }^{8,9}$. As stocks run down, approved suppliers attempt to scale up production but soon reach capacity, and other avenues are pursued to meet demand ${ }^{10}$.
In some cases, stockpiles of equipment that have reached their expiry date are pressed into service once performance has been validated ${ }^{11}$. Another valuable approach is the adaption of manufacturers with 
suitable infrastructure, switching from normal 'peacetime' production lines to emergency manufacturing in response to a pandemic. Examples we have seen during COVID-19 include cosmetic companies and distilleries switching to the production of hand sanitizers rather than perfume and alcoholic beverages ${ }^{12}$, textile companies making medical gowns and face masks ${ }^{13}$, and automotive, electronics or appliance manufactures making ventilators ${ }^{14}$. Similarly, the scale of testing could be increased if infrastructure were available that could be redeployed from peacetime applications to emergency protocols at a time of need. A similar approach was proposed by Nobel laureate Paul Nurse (Francis Crick Institute, London, UK), who used the Dunkirk troop evacuation metaphor to suggest marshalling the resources of many small laboratories (small boats) to meet testing needs ${ }^{15}$. One drawback is that the many different laboratories would require a huge effort in coordination and standardization. Instead of many small boats, we suggest a mobilization of large ships - specifically, by exploiting the capacity of high-throughput agtech laboratories.

\section{Big ships with big guns}

Dual-purpose infrastructure is an attractive option because it avoids several drawbacks of constant pandemic preparedness, such as the need for a huge stockpile of equipment and reagents with finite shelf lives and associated storage costs, the need for infrastructure that stands idle most of the time, the need to retain and train staff, and the need for regular 'war games' to test response capability. Agtech infrastructure for crop and animal breeding, crop disease management and seed testing provides not only the equivalent of peacetime infrastructure that can be redeployed to emergency biomedical use in a pandemic situation, but also the capacity to handle future outbreaks more effectively. We should develop contingency protocols for such facilities allowing them to switch to pandemic response mode.

High-throughput laboratory-based screening first emerged with plant breeders like Downey and Stefansson, who screened tens of thousands of seed samples in the 1960s to produce modern canola varieties. In the modern era, high-throughput screening has become a routine breeding approach, as seen with Monsanto's strategy to separate diploid and haploid maize seeds based on their differing oil content ${ }^{16}$. Agtech entered the digital era of high-throughput screening more than a decade ago, with the realization that a combination of remote and in-field sensing, high-throughput data analysis and confirmatory molecular testing (PCR, DNA sequencing, antibody-based detection and liquid chromatography-mass spectrometry) could be used for in-field plant phenotyping and input allocation, disease management, molecular breeding and the development of new cultivars ${ }^{17}$. Advances in medical applications have seen parallel advances in agriculture, such as the rapid adoption of genomics, transcriptomics, proteomics, metabolomics, gene editing, bioinformatics, cell-based assays and high-content image analysis ${ }^{18}$.

It is paramount that we recognize that pandemics and epidemics are not limited to humans, but can also affect other animals and plants ${ }^{19,20}$. There is often a severe impact on food security and human health, as historically seen in the famines caused by potato blight (Phytophthora infestans $)^{21}$ in the 1840 s and the epizootic rinderpest virus ${ }^{22}$ in the 1890s. The Great Famine caused by potato blight in Ireland (1845-1849) led to one million deaths and caused another million people to emigrate, and the spread of potato blight in Europe claimed another 100,000 lives. The introduction of rinderpest virus to sub-Saharan Africa was even more devastating, killing $~ 90 \%$ of infected cattle as well as many sheep, goats and the oxen used by farmers for ploughing, and also killing off swathes of wild buffalo and wildebeest, leaving no animals to farm, herd or hunt. The resulting famine killed millions, including one-third of the population of Ethiopia and two-thirds of the Maasai. The loss of grazing animals also changed the grasslands into thickets that allowed the tsetse fly to breed, resulting in a more human deaths caused by sleeping sickness ${ }^{23}$. A more recent example of epizootic disease was an outbreak consisting of more than 2,000 cases of foot and mouth disease in the United Kingdom in 2001, which prompted the slaughter of more than six million cattle and sheep at a cost of over $\$ 11$ billion $^{24}$.

Agtech infrastructure used to facilitate plant breeding and biosecurity surveillance could easily be adapted for medical screening (Fig. 1). However, the scale of routine agtech is much larger than standard healthcare screening because plant breeding involves hundreds of thousands of samples that must be processed rapidly to accommodate at least two and up to three cycles of selection based on genotyping and phenotyping per year, following approaches such as Borlaug's shuttle breeding strategy ${ }^{25}$. Furthermore, the use of agtech as part of an integrated biosecurity program to monitor pests and diseases in the field provides a high degree of overlap in terms of equipment, reagents and expertise with the resources needed to manage human epidemics and pandemics. For example, surveillance programs are in place and constantly active to monitor animal diseases such as African swine fever. This disease has broken out in China and devastated their domestic pork production industry ${ }^{26}$, and entry into North America would have devastating consequences ${ }^{27}$. The testing capacity built into these programs could be switched over to monitor human diseases in the event of an epidemic or pandemic outbreak. This would be one way to bring big ships rather than small boats to the international effort against pandemic human diseases.

\section{An agtech contingency}

Organizations such as the Global Institute for Food Security (GIFS) occupy a particular niche in the agtech ecosystem that provides an opportunity to capitalize on infrastructure and capacity in the event of a human pandemic. For example, the GIFS Omics and Precision Agriculture Laboratory (OPAL) is a state-of-the-art laboratory that provides genomics, phenomics and bioinformatics services to public and private research and commercial organizations in North America. The infrastructure at OPAL combines remote imaging and in-field environmental monitoring with genetic information to provide a complete profile of plant samples, followed by data analysis and decision-tree analytics to monitor diseases or identify genetic factors that confer beneficial phenotypes. This infrastructure can accommodate 3,000 samples per day and is largely automated, which provides plug-and-play convenience that would allow switchover to the analysis of human blood samples or swabs if health testing centers reached capacity (Fig. 1). In the case of virus testing, minimal changes to protocol would be required because the OPAL routinely screens nucleic acid samples in high-throughput assays of plant material, so a modified extraction protocol and the use of different primers for amplification would be sufficient. Routine diagnostics for COVID-19 only require biosafety level 2 facilities, with additional restrictions necessary only when the aim is to culture live virus ${ }^{28}$. For protein tests, it would be necessary to access new reagents, such as specific detection antibodies, but other reagents and components would be the same as used to detect plant proteins. GIFS has already donated materials and supplies to the local health authority, as well as lending equipment to enable the expanded testing of COVID-19 samples.

We are therefore calling for a national or international effort to develop contingency plans for human testing based on available agtech infrastructure. This would facilitate 
a much more rapid and coordinated response to testing than witnessed in response to COVID-19, particularly given that stay-at-home orders would otherwise require the laboratories to be shut down so their capacity would be largely wasted. Such an effort would require a clear chain of command to determine how the surge capacity provided by agtech would align with existing healthcare capacity and to coordinate their roles in the work flow to maximize the shared resources. Some countries, such as Australia and New Zealand, have proactively built their biosecurity programs and clearly defined networks across the country. In Australia, the Commonwealth Scientific and Industrial Research Organisation (CSIRO) is responsible for bio-vigilance and training through the Australian Centre for Disease Preparedness. Countries that focus proactively on biosecurity have been some of the better responders to the current crisis, and a similar model should be followed for the coordination of agtech infrastructure during a future pandemic response.

Ideally, each agtech institute would have a dedicated quality assurance/quality control (QA/QC) coordinator who would be responsible for International Organization for Standardization (ISO) certification, good laboratory practice (GLP) accreditation and the training of key personnel. This role could also double as a pandemic coordinator, whose job would be to oversee the switchover to pandemic preparedness in response to a disease outbreak affecting humans, other animals or plants as needs arise, to ensure personnel are trained in new processes and procedures, and to align with regulatory requirements (Fig. 1). This individual would have access to the institutional contingency plan and would know which materials and personnel to bring in to run the emergency operations while other parts of the institute were closed down or reduced to skeleton staff and essential needs only. In peacetime, the QA/QC coordinator would organize annual training for the switchover personnel, ensure that new methods are adopted and can be scaled up, maintain essential supplies, and liaise with local and national government bodies to ensure that related issues such as GLP-level documentation, data handling, privacy and security are adequate for the transition to an emergency role.

We must learn the lessons of COVID-19 and move from a reaction stance to one of preparedness. In this respect, the concept of an agtech pandemic response contingency represents an opportunity for the world to respond with alacrity to the next pandemic.

\section{Editorial note: This article has been peer} reviewed.

\section{Steven R. Webb (D) ${ }^{凶}$, Richard M. Twyman (D) and Maurice Moloney ${ }^{3}$ \\ ${ }^{1}$ Global Institute for Food Security, University of Saskatchewan, Saskatoon, Saskatchewan, Canada. \\ ${ }^{2}$ TRM Ltd, Scarborough, North Yorkshire, UK. ${ }^{3}$ AgritecKnowledge LLC, Royal Wootton Bassett, Wiltshire, UK. \\ $凶_{e-m a i l: \text { steven.webb@gifs.ca }}$}

Published online: 6 August 2020

https://doi.org/10.1038/s41587-020-0654-5

References

1. Sørensen, M. D. et al. Ann. NY Acad. Sci. 1067, 500-505 (2006).

2. de Groot, R. J. et al. J. Virol. 87, 7790-7792 (2013).

3. Johns Hopkins University \& Medicine. Coronavirus resource center. https://coronavirus.jhu.edu (accessed 30 July 2020).

4. Rowan, N. J. \& Laffey, J. G. Sci. Total Environ. 725 , 138532 (2020).

5. Swaminathan, A. et al. Emerg. Infect. Dis. 13, 1511-1547 (2007).

6. Mitchell, R. et al. Am. J. Infect. Control 40, 611-616 (2012).
7. France 24. Solidarity? When it comes to masks, it's every nation for itself. https://www.france24.com/en/20200403-solidarity-whe n-it-comes-to-masks-it-s-every-nation-for-itself (2020).

8. Carias, C. et al. Clin. Infect. Dis. 60, S42-S51 (2015). (Suppl. 1).

9. Patel, A. et al. Health Secur. 15, 244-252 (2017).

10. Mehrotra, P., Malani, P. \& Yadav, P. Personal protective equipment shortages during COVID-19-supply chain-related causes and mitigation strategies. JAMA Network https://jamanetwork.com/ channels/health-forum/fullarticle/2766118 (2020).

11. Perkins, D. J. et al. Exp. Biol. Med. (Maywood) https://doi. org $/ 10.1177 / 1535370220925768$ (2020).

12. Ledsom, A. Across the U.S. and Europe, alcohol and perfume brands ramp up the manufacture of hand sanitizer. https://www. forbes.com/sites/alexledsom/2020/03/21/across-the-us-andeurope-alcohol-and-perfume-brands-ramp-upthe-manufacture-of-hand-sanitizer/ (21 March 2020).

13. Ahmad, Z. Flint makers band together to provide masks for healthcare workers. https://www.mlive.com/coronavirus /2020/04/flint-makers-band-together-to-providemasks-for-healthcare-workers.html (10 April 2020).

14. Davies, R. UK manufacturers to regear factories to build ventilators for NHS. https://www.theguardian.com/business/2020/ mar/17/uk-manufacturers-regear-factories-build-ventilators-nhs (17 March 2020).

15. Mason, R. Boris Johnson restates pledge to boost UK coronavirus testing capacity. https://www.theguardian.com/politics/2020/ apr/02/boris-johnson-restates-pledge-toboost-uk-coronavirus-testing-capacity (2 April 2020).

16. Melchinger, A. E., Schipprack, W., Würschum, T., Chen, S. \& Technow, F. Sci. Rep. 3, 2129 (2013).

17. Shakoor, N., Lee, S. \& Mockler, T. C. Curr. Opin. Plant Biol. 38, 184-192 (2017)

18. Van Emon, J. M. J. Agric. Food Chem. 64, 36-44 (2016).

19. Almeida, R. P. P. PLoS Biol. 16, e2007020 (2018).

20. Thompson, R. N. \& Brooks-Pollock, E. Philos. Trans. R. Soc. Lond. B 374, 20190038 (2019).

21. Fry, W. E. \& Mizubuti, E. S. in The Epidemiology of Plant Diseases (ed. Jones, D. G.) 371-388 (Springer, 1998).

22. Phoofolo, P. Past \& Present 138, 112-143 (1993); https://doi. org/10.1093/past/138.1.112

23. Van den Bossche, P., de La Rocque, S., Hendrickx, G. \& Bouyer, J. Trends Parasitol. 26, 236-243 (2010).

24. Knight-Jones, T. J. D. \& Rushton, J. Prev. Vet. Med. 112, 161-173 (2013).

25. Schneider, C. CSA News 59, 4-11 (2014).

26. Wang, T., Sun, Y. \& Qiu, H.-J. Infect. Dis. Poverty 7, 111 (2018).

27. Sánchez-Cordón, P. J., Montoya, M., Reis, A. L. \& Dixon, L. K. Vet. J. 233, 41-48 (2018).

28. CDC. Biosafety for specimen handling. https://www.cdc.gov/ coronavirus/2019-ncov/lab/lab-biosafety-guidelines.html (2020).

\section{Acknowledgements}

Funding provided by the Global Institute for Food Security. S.R.W. is grateful for insightful comments and suggestions from Lorne Babiuk.

Competing interests

The authors declare no competing interests. 